\title{
Elucidating the Underlying Physical, Chemical and Biological Mechanisms for the Altered Plant uptake and Accumulation of Heavy Metals by Engineered Nanoparticles
}

\author{
Xingmao Ma \\ Texas A\&M University \\ USA
}

Prevalent heavy metal contamination in the environment has raised serious food safety concerns due to their potential accumulation in food crops. With a growing interest in the applications of nanotechnology in the foodenergy-water nexus, elevated concentrations of engineered nanoparticles (ENPs) in agricultural soils are increasingly detected. These ENPs possess very large specific surface area and uniformly decorated surface properties, and interact with a variety of co-existing environmental pollutants closely. Many previous studies have demonstrated that co-present ENPs alter the plant uptake and accumulation of heavy metals. They also modify the in-planta distribution and speciation of redox-sensitive heavy metals such as arsenic. We investigated the underlying mechanisms leading to changes in the fate and transport of heavy metals in a plant system by ENPs. In a co-contaminated site with both heavy metals and ENPs, plants tend to release higher amount of root exudates, resulting in different $\mathrm{pH}$ and $\mathrm{Eh}$ conditions in root rhizosphere. The excretion of root exudates also affects the adsorption of heavy metals on ENPs, and ENP aggregation and stability. In addition, plant roots adjust anatomically in the co-presence of ENPs and heavy metals by modifying the formation of root apoplastic barriers. These different processes together govern the fate and uptake of heavy metals by plants in a co-contaminated site with ENPs. 